\title{
Towards Night Fog Detection through use of In-Vehicle Multipurpose Cameras
}

\author{
Romain Gallen, Aurélien Cord, Nicolas Hautière and Didier Aubert
}

\begin{abstract}
Compared to daytime, a larger proportion of accident happens during nighttime. The altered visibility of the road scene for the drivers may partially explain this situation. The latter becomes worse in fog presence. In this paper, two camera-based methods are proposed to detect the presence of night fog in images grabbed by in-vehicle multipurpose cameras. They rely on the visual effects of night fog. A first approach can assess the presence of fog around the vehicle thanks to the detection of the backscattered veil induced by the vehicle ego lights. It aims at detecting fog when the vehicle is alone in absence of exterior public lighting. A second approach can assess the presence of fog thanks to the detection of halos around light sources in the vehicle environment. It aims at detecting fog in presence of road traffic or public lighting. Both methods are presented and illustrated with actual images of fog. Their complementarity makes it possible to envision a complete night fog detection system. There are numerous applications for such a system: automation or adaptation of vehicle lights, contextual speed computation and reliability improvement for camera-based systems.
\end{abstract}

\section{INTRODUCTION}

While night driving represented only $10 \%$ of total traffic, it caused 44\% of fatalities in 2006 in France. Moreover, accident severity during nighttime increases by a factor of 1.7 as compared with daytime [1]. Among the factors which explain these figures, drowsiness [2], speed and reduced visibility are frequently cited. To address this issue, emphasis is put on the development of smarter lighting strategies for the vehicles [3]. Systems are being developed to automate and adjust the operation of vehicle lights so as to prevent glaring oncoming drivers. Automotive lighting is thus becoming a new branch of Intelligent Vehicles activities.

Among the embedded sensors, video cameras are among the most promising ones since they can be low cost and can address different issues. In particular, degraded weather conditions such as rain or fog, are major concerns. First, adverse weather conditions directly affect the safety of the driver by reducing his safety margin: low visibility distance or low friction. Second, the reliability of camera-based systems is reduced due to altered image quality. For these two reasons, detecting, characterizing and mitigating the effects of fog by camera is a challenge for camera-based ADAS.

It is noticeable that the automation and the automatic adaptation of the intensity of lights in fog is still an open issue though regulations allows it [4]. Fog is known for its effects

R. Gallen (current affiliation: CETMEF) and A. Cord are with UniverSud, LIVIC, IFSTTAR, bldg 824, 14 route de la Minière, 78000 Versailles, France e-mail: aurelien.cord@ifsttar.fr.

N. Hautière and D. Aubert are with the Universite Paris-Est, LEPSIS, IFSTTAR, 58 boulevard Lefebvre 75015 Paris, France e-mail: nicolas.hautiere@ifsttar.fr, didier.aubert@ifsttar.fr. on visibility but the visual effects of fog differ between night and day, which explains why camera-based detection methods also differ. On-board daytime fog detection has been pioneered in [5] and further tackled in [6]. Mitigation of daytime fog has been addressed by restoring the contrast of images plagued by fog [7]. The case of heterogeneous daytime fog has also been addressed in [8].

In this paper, the problem of on-board night fog detection by camera is addressed. Contrary to daytime, at night, the visual appearance of a road scene in presence of fog depends strongly on the presence of artificial light sources in the environment. If a vehicle is alone on the road and there is no public lighting installation, the fog can only be noticed by the backscattering of the lights of the ego vehicle. If the vehicle is crossing other vehicles or if there is a public lighting installation, the backscattered veil is no longer noticeable but the presence of fog can still be noticed thanks to the presence of halos around the light sources. Consequently, a relevant night fog detection system must be able to deal with these two types of lighting environments. We have developed two algorithms, each of them is dedicated to a particular environment. To our best knowledge, there exist no publications dealing with this problem. However, different recent industrial patents have been deposited and are now discussed.

For night fog detection, some static camera-based systems rely on the comparison from the same point of view of images containing light sources [9], [10], [11], [12] or specific high contrasted targets [13]. But those methods can only been used in a static way and cannot be adjusted to dynamic conditions such as driving. Among the existing ADAS, some systems use the blurring of the tail lamps of a leading vehicle to determine the presence of fog according to the degree of blur of the tail lamps, like in patent JP 11$278182 \mathrm{~A}$. This does not work anymore if there is no vehicle ahead of the equipped vehicle. The two patented camerabased system [14], [15] rely on the backscattered light of headlamps in order to detect the presence of fog. They both proceed by analyzing the evolution of pixel intensities along a vertical or horizontal line in the image. Those local methods may be impaired by the presence of obstacles or markings in the scene and because the backscattered light energetic level is quite low, their behavior may also be impaired when there are multiple light sources in the environment, such as roads lighted with street lights or with oncoming traffic. Unlike patent JP 11-278182A, our method does not require other cars or obstacle to be in front of the equipped vehicle in order to work. Unlike patents [14], [15] 
that may not work in strongly lit environments, our dual system is able to deal with all sorts of lighting environments and do not use a local or photometric approach but a global image analysis process. A patent is deposited for our dual system under number INPI-1057802.

There are numerous applications for such a system: automation or adaptation of vehicle lights, contextual speed computation [16] and camera-based system reliability improvement. This paper is organized as follows. The visual effects of night fog are detailed first. The detection of the backscattered veil is then carried out. The detection of halos around light sources follows. Finally, results are discussed and perspectives for future research are given.

\section{Visual EFFECTS OF Night Fog}

Fog is a cloud on the ground. It is composed of billions of tiny water droplets floating in the air. The visibility loss results from airborne droplets which scatter light, causing drastic alterations in the image of the environment perceived by vision systems, both human and artificial [17]. The amount of scattering is characterized by a coefficient $k$. The meteorological visibility distance $V_{\text {met }}$, commonly used to describe the fog density, is conversely proportional to $k$ [18]. Scattering results in a reduction of the perceived luminances and has two major visual effects in nighttime. First, when driving in fog, light from the headlamps is back-scattered toward the driver, producing an additional veil in the driver's field of vision as schematized in Figure 1(a). This phenomenon is always present. However, it is no longer visible in presence of external light sources like public lighting or other vehicles lights. In such case, according to the atmospheric modulation transfer function (MTF) theory, the resulting halo (or glow) effect is equivalent to the convolution of the light source image with the point spread function (PSF) of the fog [17]. This effect is schematized in Figure 1(b).

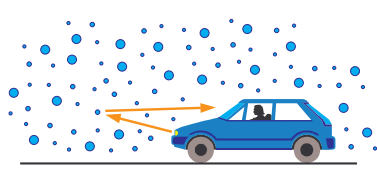

(a)

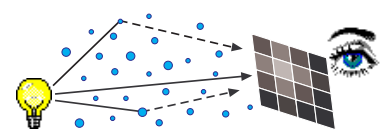

(b)
Fig. 1. Visual effects of fog in night driving: (a) backscattering of the ego-lights of the vehicle; (b) halo around artificial light sources.

Based on these two visual effects, a system composed of two detection algorithms has been developed. The first detection system aims at detecting the presence of a backscattered veil. The second detection system aims at detecting the presence of halos around artificial light sources in the environment.

\section{Detection of the Backscattered Veil}

The first system is based on the fact that light emitted from a vehicle's lighting system is scattered backwards toward the observer in the presence of fog. The idea investigated to detect it is to compare different reference images with the current image grabbed by the camera using a correlation index.

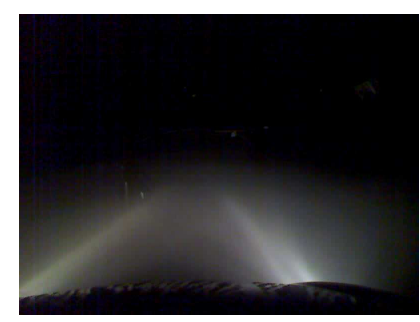

(a)

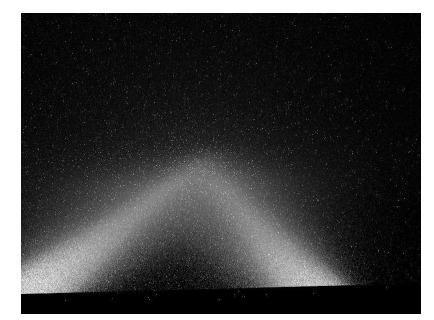

(b)
Fig. 2. Backscattered veil: (a) actual images grabbed in the ClermontFerrand fog chamber [19]; (b) simulated image using semi Monte Carlo raytracing software [20].

The reference image represents the front of the vehicle with lighting on as can be seen in fog on a dark road. It could be a real image taken with the same camera in a real situation of fog or a synthetic image generated in order to simulate the on-board camera. The process allowing to generate such an image can be of any type. For instance, it could rely on a Monte Carlo method allowing the synthesis of images in fog or standard image processing techniques allowing for fog rendering in 3D scenes (see Figure 2(b)) [20]. When generating a synthetic image, this reference image should be produced knowing the type and position of the car lamps, the positioning of the camera inside the car including its relative angle as compared with the axis of the car. Moreover, the focal length and the image resolution of the camera should also be known in order to generate an image as close as possible to a real image acquired with the camera. Several reference images may be computed or acquired in this way with different fog densities or fogs with different size distributions of droplets.

These reference images are then compared with the current image (see Figure 2(a)) through means of image correlation techniques such as: SAD (Sum of Absolute Differences), ZSAD (Zero mean Sum of Absolute Differences), SSD (Sum of Squared Differences) ZNSSD (Zero mean Normalized Sum of Squared Differences), or any distance related measure between two images. The used synthetic images generator produces luminance maps. Converting the range of illuminations into 8 bit images is done by clipping lowest illuminance values to 0 and highest illuminance values to 255 . Moreover, the exposure settings of the camera are unknown. Synthetic and real images may have different dynamics. Among available correlation distances, ZNSSD has been chosen for its invariant properties regarding mean and scale of illumination level. This method gives reliable correlation index and presents the best results for fog discrimination task.

This comparison score can be computed using whole initial image and whole reference image or it can also be computed on a smaller part of the images. Indeed, both whole images around the backscattering veil are very dark. It induces a high correlation score whatever the fog density and then decreases the discrimination score. To overcome this 
problem, a mask image corresponding to the backscattering veil is produced using synthetic images with varied fog intensities. The correlation index is only evaluated inside this mask.

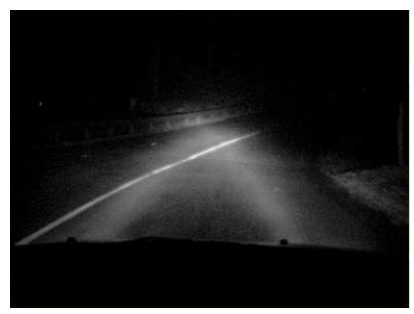

(a)

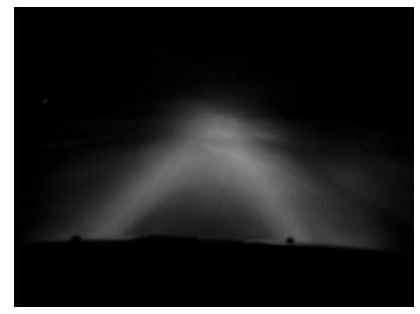

(b)
Fig. 3. Actual fog sequence grabbed during a test drive: (a) sample image; (b) mean of successive images. The object in the road scene are suppressed whereas the backscattered veil is highlighted thanks to its temporal stability.

The initial image of the front of the vehicle can either be the current image acquired from the camera or an image that is the composition of successive images due to the temporal stability of the visual effect with time. The averaged image can be computed by different means of averaging an image like mean of pixel values across $n$ successive images, a rank filter like the median filter on the pixels of $n$ successive images (see Figure 3). As one can see, the backscattered veil is kept in the image whereas other objects like lane markings are likely to disappear.

By applying a threshold on the correlation score we can confirm the presence of fog. High correlation scores between a synthetic image with fog and the image from the camera in the vehicle correspond to the presence of fog while low correlation scores correspond to the absence of fog. When using several reference images, we get several comparison scores with the initial image, the higher score among them can be selected for fog detection. To validate our method, we have recorded videos of fog in the ClermontFerrand fog chamber (France) [19] where we varied the fog density. Thanks to the monitoring devices (in particular a transmissometer) installed in the room, we are able to check the output of the algorithm. Part of the reference image database used in the test is shown in Figure 4. A sample of result is given in Figure 5 for the ZNSSD correlation metric. As one can see, for a given reference image the highest correlation score is obtained when the actual meteorological visibility distance is close to the simulated one.

\section{Detection of Halos around Light Sources}

When light sources appears in the road environment, the backscattering veil becomes undetectable due to automatic camera settings and reduced camera dynamic. However, as discussed in section II, in night fog, halos are present around light sources. A halo is the consequence of light scattering induced by water droplets. In the image, it corresponds to an intensity decrease as the distance from the source increases. Its expanse and its intensity decrease depend mainly on the fog density and droplet sizes. In the following, the algorithm aiming at detecting and characterizing halos in the image is

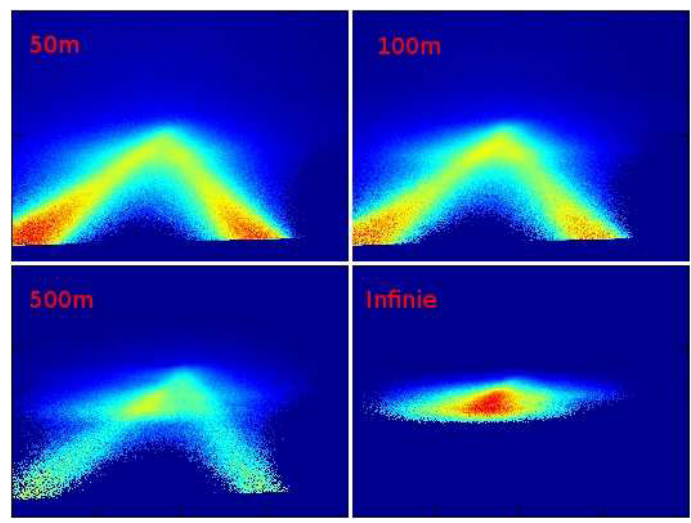

Fig. 4. Database of simulated images with different fog densities and consequently different meteorological visibility distance. Color scale goes from blue to red, in order to bring out intensities variations.

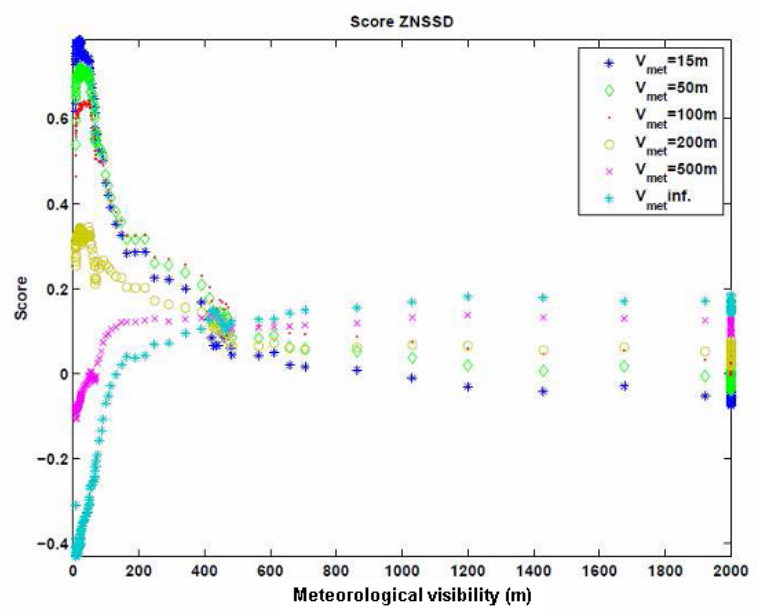

Fig. 5. ZNSSD correlation score between a reference image and a fog image grabbed during a fog dissipation in the fog chamber of ClermontFerrand.

presented. Then, an evaluation of the approach is proposed on a video database recorded during foggy and clear nights.

The algorithm is decomposed in the following steps:

- Light sources detection. It is based on an image thresholding at a high intensity level, close to the maximum value of image pixels. It is tuned to segment at least $3 \%$ of the image. Each connected component is extracted and some geometrical parameters are evaluated: position of the center of gravity, surface, compactness, elongation. Relying on those parameters, connected components are selected in order to exclude those that do not correspond to isolated source. For instance, lane marking are excluded relying on its geometrical pattern and some sources, that are agglomerated due to their proximity in the image, are excluded relying on their large surface. The selected connected components are called "potential sources". As illustrated in Figure 6, we start to build a tree. The leafs are the potential sources associated with their geometrical parameters.

- Halo segmentation. By progressively decreasing the 


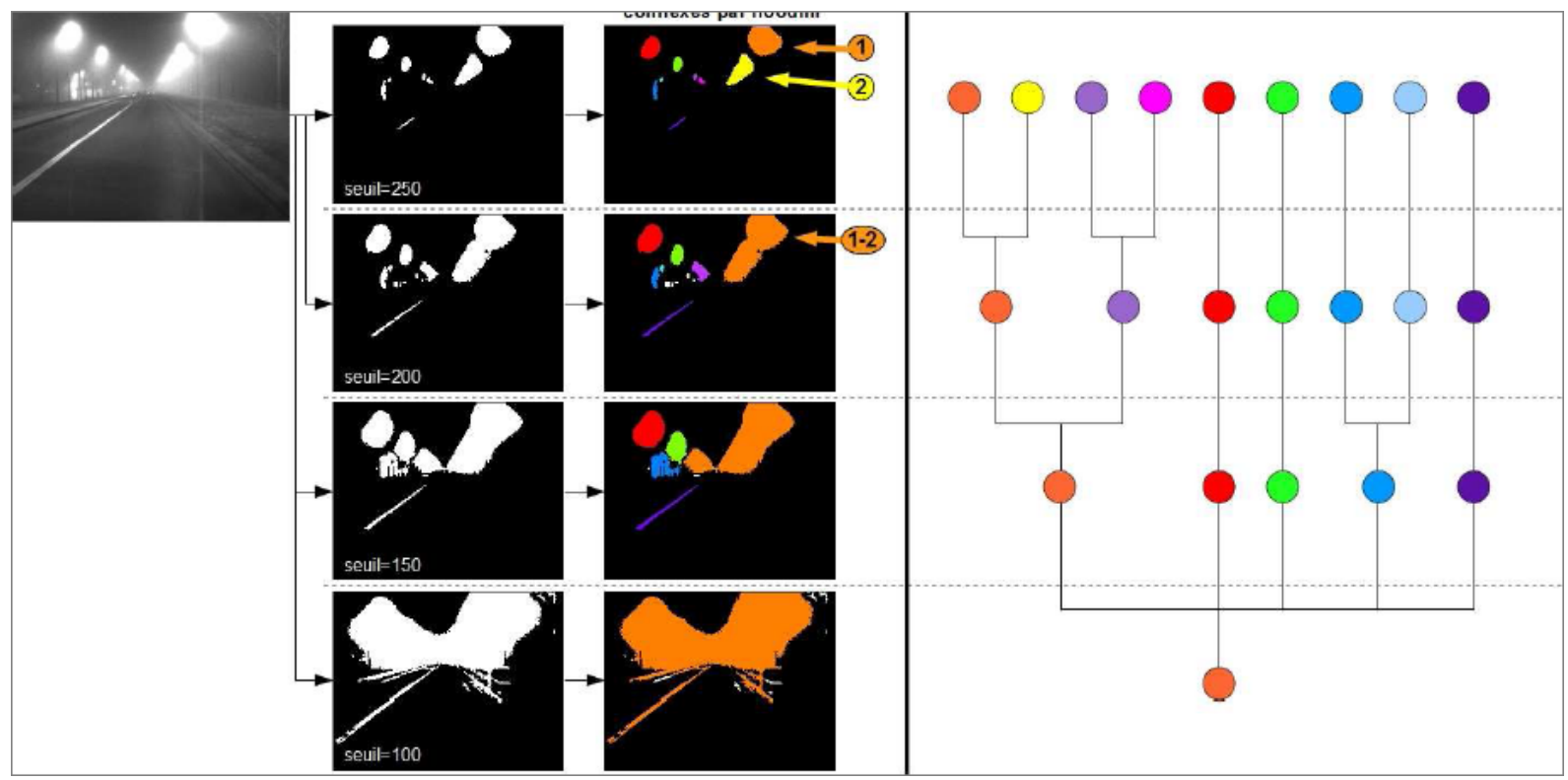

Fig. 6. Detection of light sources and associated halos. On the left, original image. In the middle, segmented images with decreasing thresholds and associated connected components. On the right, the tree built using the segmented images.

threshold, a segmented connected component should contains a larger part of each source halo in addition to the source itself. For each smaller threshold, the connected components are extracted using the potential sources as a seed. A new node is added to the tree, under the corresponding potential source's leaf, containing the same geometrical parameters. If two or more potential sources belong to the same connected component, their branches are merged in the same node. We continue the threshold decrease until all the potential sources merge. Notice that we do not add new leaves to the tree during this process.

- Halo selection. Based on the geometrical parameters, calculated at each step, we exclude the branches for which :

- the size growth between two adjacent thresholds is large, typically more than 5 times. It corresponds to the fusion of the potential source with a bright object present in the scene.

- the gravity centers evolve drastically without a preferred direction. It generally do not correspond to sources.

- Determination of halo direction. Each branch of the builded tree is studied independently and the Center of Gravity (CoG) of the segmented shapes are computed for each node. A linear regression on the successive positions of the $\mathrm{CoG}$ along a branch gives the direction of evolution of the halo. If a source is isotropic (all CoGs are merged) an arbitrary direction is chosen.

- Halo intensity profile extraction. The intensity profile is extracted along the direction estimated in the previous step, beginning at the $\mathrm{CoG}$ of the source segmented in step 1 (Light sources detection). Figure 7 illustrates how crucial is the direction choice. For directional light sources, the halo is only present in the lighting direction (green direction in this case).
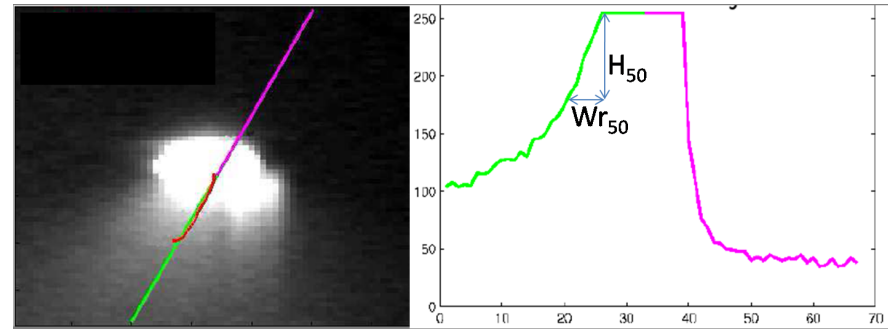

Fig. 7. Intensity evolution of a halo.

- Characterization of the intensity profile of the halo. In presence of fog, the decrease of intensity, from the center to the outside of a light source, is smoother than in absence of fog. This characteristic enables to detect fog presence. The choice of the curve characteristic is crucial. A large set of characteristics were explored and compared in a statistical point of view in order to select the most relevant one to distinguish between the presence and the absence of fog.. As illustrated in Figure 7 , the following curve characteristic is chosen: $B r_{50}=$ $H_{50} / W r_{50}$, with $H_{50}$ half the height of the decrease and $W r_{50}$ the width between the end of the sensor saturation and the curve point corresponding to the half of the height. $B r_{50}$ decreases in presence of fog.

- Br50 combination. Each image of our video sequence contains several potential sources. The characteristics of the halos are then combined by averaging all $\mathrm{Br}_{50}$ characteristics extracted from potential sources.

Four video sequences were produced using an embedded camera on the same location at night: 2 of them during clear nights and the other 2 during foggy nights. They present 

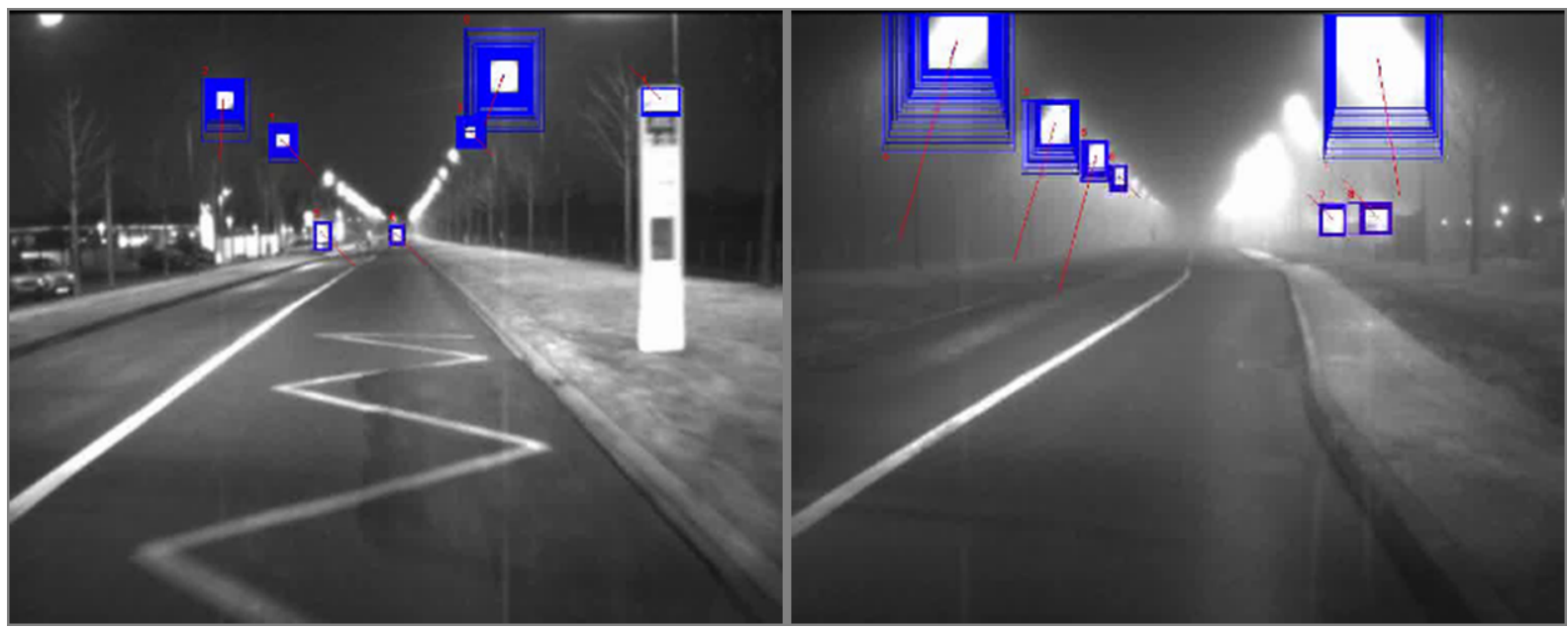

Fig. 8. Illustration of source halos detection. On the left, in absence of fog. On the right, in presence of fog. Blue boxes represent the connected components bounding boxes at each step of potential sources.

2130 images which contain an average of 10 light sources per image. Figure 8 illustrates the source and halo detection on 2 images extracted from the database.

Considering each image independently, the proposed algorithm produced a fog detection rate of $98.4 \%$.

By producing a sliding average of the characteristic of the halo profile on 1 second of video ( 25 images), the fog detection rate reaches $99.86 \%$.

\section{Discussion And Perspectives}

The two algorithms, coded in $\mathrm{C}++$ using OpenCV library, runs in real-time (25 images per second) on a typical desktop PC. An implementation on micro-controller could be considered in order to have an advanced driving assistance system.

In classical driving condition during night time, the car is alone on the road without any artificial light sources. In this case the detection of backscattered veil provides the information of the fog presence. As soon as artificial light sources appear in the camera field of view, this veil becomes undetectable due to both the low dynamic range and the automatic setting of the camera. The detection of halos around light sources takes over and provides the fog presence information. The combination of these two algorithms gives a fully functional system for fog detection at night by an embedded camera.

The two algorithms give both very good results in term of fog detection on our video database. However, a larger video database is needed to fully evaluate the performance. In particular, we plan to study the response of the algorithms for different fog intensities and natures (fogs composed of different distributions of droplet size diameters produce different visual effects), for various camera settings and in different road scene environments (for instance, vehicle crossing). Moreover, the transition between the two algorithms needs to be studied more deeply.

Such a database, associated with a ground truth on the fog density and nature, could be used to improve both algorithms in order to go from fog detection to fog characterization. Indeed, we could search for indicators that are linked with the fog properties. If so, such a system could be used to evaluate the meteorological optical range. At night, this value corresponds to the distance at which a collimated beam of light is attenuated of $95 \%$. This important perceptual and meteorological parameter depends on fog density as well as on the droplets size distribution and it has been shown that this last aspect of fog is not to be neglected in the models [12].

\section{CONCLUSION}

The challenge here was to provide a method allowing for fog detection using a standard camera with automatic exposure settings. No adjustment for the specific purpose of detecting fog at night has been done. This choice was made to preserve the usual working state of other camerabased ADAS. This strategy implied the development of a dual algorithm that stands for situations when the vehicle is only lit by his headlights and for situations when the environment is lit by multiple light sources.

Three main fields of the ITS are aimed to:

- Driver/car orientated ADAS: this dual algorithms allows us to achieve realistic and usable night fog detection method. This method combined with previous works cited in section I provides the first complete camera-based system allowing to detect the presence of fog in most conditions (day/night, dark/lit environment). This could be used in order to inform the driver on his visibility loss and to automatically turn on/off fog lights or to adapt the intensity and directionality of the front and rear-lights as proposed in [21].

- Safety orientated ADAS: the detection of fog could be used in a wider safety system such as a dynamic intelligent speed adaptation system as proposed in [16]. Provided that vehicular/infrastructure communications 
are available, such a system could also be used in a global information system in order to warn drivers before they enter a fog cloud. It also could be exploited to plan a route taking into account both traffic and meteorological information.

- System orientated ADAS: usual computer vision methods dedicated to ADAS such as lane detection, obstacle detection, free road space detection are usually unable to simultaneously provide an information on their current working status. Worse, even their inventors do not know most of the time what could be the impact of fog on such systems (more false negatives, more false positives, etc.). Detecting fog is thus an unavoidable tool that could be used to negate or to mitigate the results of other algorithms.

Finally, the detection of fog at night open new field of research : image restoration under night fog situations. This could be a way to enhance the perception of the driver in fog and to compensate for image degradations induced by fog presence in order to preserve the normal behavior of other camera-based ADAS.

\section{ACKNOWLEDGMENTS}

This work is partly funded by the ANR (French National Research Agency) ICADAC project (6866C0210).

\section{REFERENCES}

[1] "Les accidents corporels de la circulation routière en 2008," Observatoire national interministériel de sécurité routière, Tech. Rep., January 2009.

[2] F. Friedrichs and B. Yang, "Camera-based drowsiness reference for driver state classification under real driving conditions," in IEEE Intelligent Vehicles Symposium, San Diego, California, USA, June 2010, pp. 101-106.

[3] M. Tideman and S. J. Janssen, "A simulation environment for developing intelligent headlight systems," in IEEE Intelligent Vehicles Sympoisum, San Diego, California, USA, 2010.

[4] Type-approval requirements for the general safety of motor vehicles, Add. 37, Rev. 2, Amend. 2, Rear fog lamps. United Nations Economic Commission for Europe, 2007.
[5] N. Hautière, J.-P. Tarel, J. Lavenant, and D. Aubert, "Automatic fog detection and estimation of visibility distance through use of an onboard camera," Machine Vision and Applications, vol. 17, no. 1, pp. 8-20, 2006.

[6] S. Bronte, L. Bergasa, and P. Alcantarilla, "Fog detection system based on computer vision techniques," in IEEE Conference on Intelligent Transportation Systems, Saint-Louis, Missouri, USA, 2009, pp. 1 -6.

[7] N. Hautière, J.-P. Tarel, and D. Aubert, "Mitigation of visibility loss for advanced camera based driver assistances," IEEE Transactions on Intelligent Transportation Systems, 2010, to appear.

[8] J.-P. Tarel, N. Hautière, A. Cord, D. Gruyer, and H. Halmaoui, "Improved visibility of road scene images under heterogeneous fog," in IEEE Intelligent Vehicle Symposium (IV'10), San Diego, California, USA, June 2010, pp. 478-485.

[9] S. G. Narasimhan and S. K. Nayar, "Vision and the atmosphere," International Journal of Computer Vision, vol. 48, no. 3, pp. 233254, 2002.

[10] S. Narasimhan and S. Nayar, "Shedding light on the weather," in Proc. IEEE Conference on Computer Vision and Pattern Recognition, 2003.

[11] S. Metari and F. Deschênes, "A new convolution kernel for atmospheric point spread function applied to computer vision," in Proceedings of the IEEE International Conference on Computer Vision, Rio de Janeiro, Brazil, October 2007.

[12] R. Gallen, N. Hautière, and E. Dumont, "Static estimation of the meteorological visibility distance in night fog with imagery," IEICE Transactions on Information and Systems, vol. E93-D (7), p. 1780:1787, 2010.

[13] T. M. Kwon, "Video camera-based visibility measurement system," Patent 7016045 , March, 2006.

[14] J. Leleve, A. Bensrhair, , and J. Rebut, "Method for detecting night fog and system implementing said method," Patent EP1 715 456, August, 2008.

[15] N. Kawasaki, T. Miyahara, and Y. Tamatsu, "Visibility condition determining device for vehicle," Patent 20080007 429, January, 2008.

[16] R. Gallen, N. Hautière, and S. Glaser, "Advisory speed for intelligent speed adaptation in adverse conditions," in IEEE Intelligent Vehicles Symposium, San Diego, California, USA, June 2010, pp. 107-114.

[17] E. Dumont, N. Hautière, and R. Gallen, Atmospheric Turbulence, Meteorological Modeling and Aerodynamics. Nova Science, NewYork, March 2010, ch. A Semi-Analytic Model of Fog Effects on Vision, pp. 635-670.

[18] CIE, International Lighting Vocabulary, 1987, no. 17.4.

[19] M. Colomb, K. Hirech, P. Andre, J. Boreux, P. Lacote, and J. Dufour, "An innovative artificial fog production device improved in the european project FOG," Atmospheric Research, vol. 87, pp. 242-251, 2008.

[20] E. Dumont, "Semi-monte-carlo light tracing for the study of road visibility in fog," in Monte Carlo and Quasi-Monte Carlo Methods 1998. Berlin: Springer-Verlag, 1999, pp. 177-187.

[21] T. Luce, "Intelligent rear lamps - a breakthrough for safety and comfort," in Proceedings of the SPIE, vol. 5663, 2005. 